\title{
Artigo/Article
}

\section{AIDS e doenças oportunistas transmissíveis na faixa de fronteira brasileira}

\author{
AIDS and transmissible opportunistic diseases in the Brazilian border area
}

\author{
Antonio Luiz Rodrigues-Júnior ${ }^{1}$ e Euclides Ayres de Castilho²
}

\begin{abstract}
RESUMO
Introdução: A transmissão do HIV e de outras doenças transmissíveis, de pessoa a pessoa, pode ser associada à mobilidade humana. Este trabalho avaliou a incidência de doenças oportunistas transmissíveis entre os casos de AIDS, nos municípios da faixa de fronteira brasileira. Métodos: Os municípios da faixa de fronteira brasileira foram agrupados em três regiões culturais; foram consideradas as notificações feitas ao Ministério da Saúde, entre 1990 e 2003, que tenham sido feitas com os critérios de definição CDC adaptado, Rio de Janeiro/Caracas e óbito; as doenças oportunistas detectadas foram agrupadas de acordo com o tipo de transmissão: 1) inalação do agente; 2) ingestão de água/alimento contaminado e 3) contato interpessoal. A análise descritiva considerou regiões culturais, anos de escolaridade, categoria de transmissão, sexo e faixa etária. Resultados: Houve diferentes padrões de incidência de AIDS nos grupos de doenças oportunistas em cada região cultural. A região extremo-sul apresentou a maior incidência de AIDS; o número de casos de AIDS do sexo feminino foi maior na categoria heterossexual; o número de casos de AIDS do sexo masculino foi maior entre usuários de drogas injetáveis; as doenças transmitidas pelo contato interpessoal foram as mais frequentes, destacando a incidência de monilíases; a tuberculose e a pneumonia foram as mais frequentes dentre as doenças transmitidas pela inalação do agente; as doenças transmitidas pela ingestão de água/alimentos contaminados mostraram um padrão de incidência estável. Conclusões: A fronteira brasileira é um espaço geográfico importante e heterogêneo; o enfrentamento da AIDS deve reconhecer as diferentes geografias culturais.
\end{abstract}

Palavras-chaves: AIDS. Faixa de fronteira brasileira. Doenças oportunistas. Geografia da fome.

\begin{abstract}
Introduction: Person-to-person transmission of HIV and other communicable diseases may be associated with human geographic mobility. This article evaluated the incidence of transmissible opportunistic diseases among AIDS cases that had been reported by municipalities in the Brazilian border area. Methods: Brazilian border area municipalities were grouped into three cultural regions; the source data was AIDS cases registered with the Ministry of Health from 1990 to 2003, which were classified according to CDC-adapted, Rio de Janeiro/Caracas and death criteria; detected communicable opportunistic diseases were categorized into groups according to transmission: 1) inhalation agent; 2) contaminated water and/or food ingestion, and 3) interpersonal contact. The descriptive evaluation considered cultural region, years of schooling, sex and age group. Results: Different AIDS incidence patterns were observed among groups of opportunistic diseases in each cultural region. The extreme southern region showed the greatest incidence of AIDS; the absolute incidence of female cases was greatest in the category of heterosexual transmission; the number of male cases was greatest among intravenous drug users; transmission was most frequent in the interpersonal contact group, particularly incidences of candidiasis; tuberculoses and pneumonias were most frequent in the inhalation agent transmission group; the contaminated water/food ingestion transmission group showed an unchanged pattern of absolute incidence. Conclusions: The Brazilian border area is a very important and heterogeneous geographic phenomenon; AIDS programs must recognize different cultural geographies.
\end{abstract}

Key-words: AIDS. Brazilian border area. Opportunistic diseases. Geography of hunger.

1. Departamento de Medicina Social, Faculdade de Medicina de Ribeirão Preto, Universidade de São Paulo, São Paulo, SP. 2. Departamento de Medicina Preventiva, Faculdade de Medicina, Universidade de São Paulo, São Paulo, SP.

Endereço para correspondência: Dr. Antonio Luiz Rodrigues-Júnior. Dept ${ }^{\circ}$ Medicina Social/FMRP/USP. Av. Bandeirantes 3900, 14049-900 Ribeirão Preto, SP, Brasil.

Tel: 55 16 3602-2537; 551636022433

e-mail: alrj@fmrp.usp.br

Recebido para publicação em 27/10/2009

Aceito em 21/07/2010

\section{INTRODUÇÃO}

A faixa de fronteira brasileira é formada por 588 municípios situados a $150 \mathrm{~km}$ da divisão geopolítica com Guiana Francesa, Suriname, Guiana, Venezuela, Colômbia, Peru, Bolívia, Paraguai, Argentina e Uruguai, formando uma região com grandes diferenças sociais, econômicas e culturais, caracterizada por conflitos armados, pelo narcotráfico, pelo contrabando, pelas atividades do crime organizado internacional, pelo desemprego, pelas grandes festas populares e pelo baixo desenvolvimento humano ${ }^{1-8}$, que, certamente, explicam as variações transnacionais do padrão epidemiológico da epidemia de HIV/AIDS ${ }^{1,9,10}$.

Figuram, dentre as doenças oportunistas transmissíveis indicativas de AIDS, a salmonelose, a toxoplasmose cerebral, a criptococose extrapulmonar, a criptosporidiose intestinal crônica e a isosporidiose intestinal crônica, como doenças transmitidas pelo contato com a fauna silvestre ou pela ingestão de água e/ou alimentos contaminados. Figura a tuberculose pulmonar, que é transmitida pela inalação do agente quando expelidos por indivíduos bacilíferos em esforços respiratórios, e a pneumonia por Pneumocystis carinii, cuja transmissão entre seres humanos não é bem conhecida, e as micobacterioses disseminadas em órgãos que não sejam o pulmão, pele ou linfonodos cervicais (exceto tuberculose e hanseníase). Figuram, também, a herpes e as monilíases, cujos agentes etiológicos são transmitidos pela saliva, e que, por representarem condições clínicas menos graves, não motivam a busca pelo atendimento médico, sendo, portanto, subnotificáveis ${ }^{11}$. Figura, ainda, a histoplasmose disseminada. As doenças emergentes, apesar de não figurarem entre as doenças indicativas de AIDS, representam perigos iminentes, como a gripe aviária, a síndrome respiratória severa aguda e a febre do Nilo ${ }^{12,13}$. No Brasil, para fins de notificação, são usados três critérios de definição de casos de AIDS estabelecidos em 2003: critério CDC adaptado, critério Rio de Janeiro/Caracas e critério de óbito.

O objetivo deste trabalho é descrever a evolução das doenças oportunistas transmissíveis associadas à AIDS, na faixa de fronteira brasileira, entre 1990 e 2003, segundo categoria de transmissão, regiões culturais, sexo, faixa etária e anos de escolaridade. 


\section{MÉTODOS}

Foi realizado um estudo ecológico de base territorial com os municípios da faixa de fronteira brasileira, de acordo com a Lei $\mathrm{n}$ ㅇ 6.634 de 1979 , que foram agrupados em regiões culturais, conforme a divisão proposta por Josué de Castro em áreas de fome ${ }^{14}$ : Amazônica, centro-oeste e extremo-sul, que são apresentadas na Figura 1. O Programa Nacional de DST e AIDS (PN-DST/AIDS) do Ministério da Saúde forneceu as informações dos casos notificados entre 1을 janeiro de 1990 e 31 de dezembro de 2003, que foram caracterizados segundo a tríade de epidemiologia, enfocando pessoa, local e tempo, além das categorias de transmissão (homens que fazem sexo com outros homens, bissexual, heterossexual, usuários de drogas injetáveis, pessoas com hemofilia, pessoas que tenham recebido transfusões de sangue, perinatal e ignorada); foram consideradas as distribuições por sexo (masculino e feminino), por faixa etária em anos $(0-13 ; 14-23 ; 24-43 ; 44-63$ e acima de 64$)$, pelo número de anos de escolaridade ( $1-3 ; 4-7 ; 8-11$ e acima de 12$)$ e por períodos trimestrais, de 1990 a 2003, em que o diagnóstico ocorreu. Optou-se por usar o número absoluto de casos notificados de AIDS e de doenças oportunistas transmissíveis, por região cultural (faixas de fronteira Amazônica, Centro-Oeste e Extremo-Sul), para manter a magnitude da epidemia, por ser uma informação interessante sob o ponto de vista do planejamento da assistência, tanto de casos notificados de AIDS quanto de doenças oportunistas transmissíveis, que, também, foram agrupadas em períodos trimestrais, em cada região cultural, que foram usadas na caracterização temporal da epidemia. Os coeficientes de incidência ${ }^{15}$ foram calculados com os tamanhos populacionais fornecidos pelo Departamento de Informática do Sistema Único de Saúde (DATASUS) para o ano de 2000.
Foram consideradas as frequências absolutas de candidíase esofágica, citomegalovirose, criptococose, criptosporidiose, herpes simples, histoplasmose, isosporidiose, pneumonia por Pneumocystis carinii, micobacteriose disseminada, salmonelose e toxoplasmose, que são doenças transmissíveis indicativas de AIDS no critério CDC-adaptado. Também foram consideradas frequências absolutas de candidíase oral, herpes zoster e tuberculose pulmonar, por serem as doenças transmissíveis indicativas de AIDS pelo critério Rio de Janeiro/Caracas ${ }^{16,17}$. Estas doenças foram agrupadas, segundo a principal via de transmissão, em: 1) doenças transmitidas pela inalação do agente patogênico, como tuberculose, micobacteriose, pneumonia, criptococose e histoplasmose; 2) doenças transmitidas pela ingestão de água e/ou alimentos contaminados, como salmonelose, criptosporidiose, isosporidiose e toxoplasmose; 3) doenças transmitidas pelo contato interpessoal, como monilíases, herpes simples, herpes zoster e citomegalovirose. Optou-se por analisar as doenças oportunistas transmissíveis, porque a faixa de fronteira representa uma região geográfica de interface entre Nações, por onde ocorrem intercâmbios entre culturas tradicionais e modernas, trocas comerciais legais e ilegais, migrações humanas temporárias e permanentes, que, além de promover o contato entre as pessoas, possibilitam a transmissão de doenças, como a AIDS.

\section{RESULTADOS}

Foram notificados 7.974 casos de AIDS na faixa de fronteira brasileira no período de estudo. A distribuição por região cultural indicou a faixa de fronteira centro-oeste com o mais alto coeficiente de incidência, apesar da faixa de fronteira extremo-sul ter notificado $72,1 \%$ do total de casos e de apresentar $96,4 \%$ da população da faixa de fronteira brasileira (Tabela 1). Do total de casos, as categorias de transmissão sexual, 71,4\% das notificações, sendo $57,7 \%$ na categoria

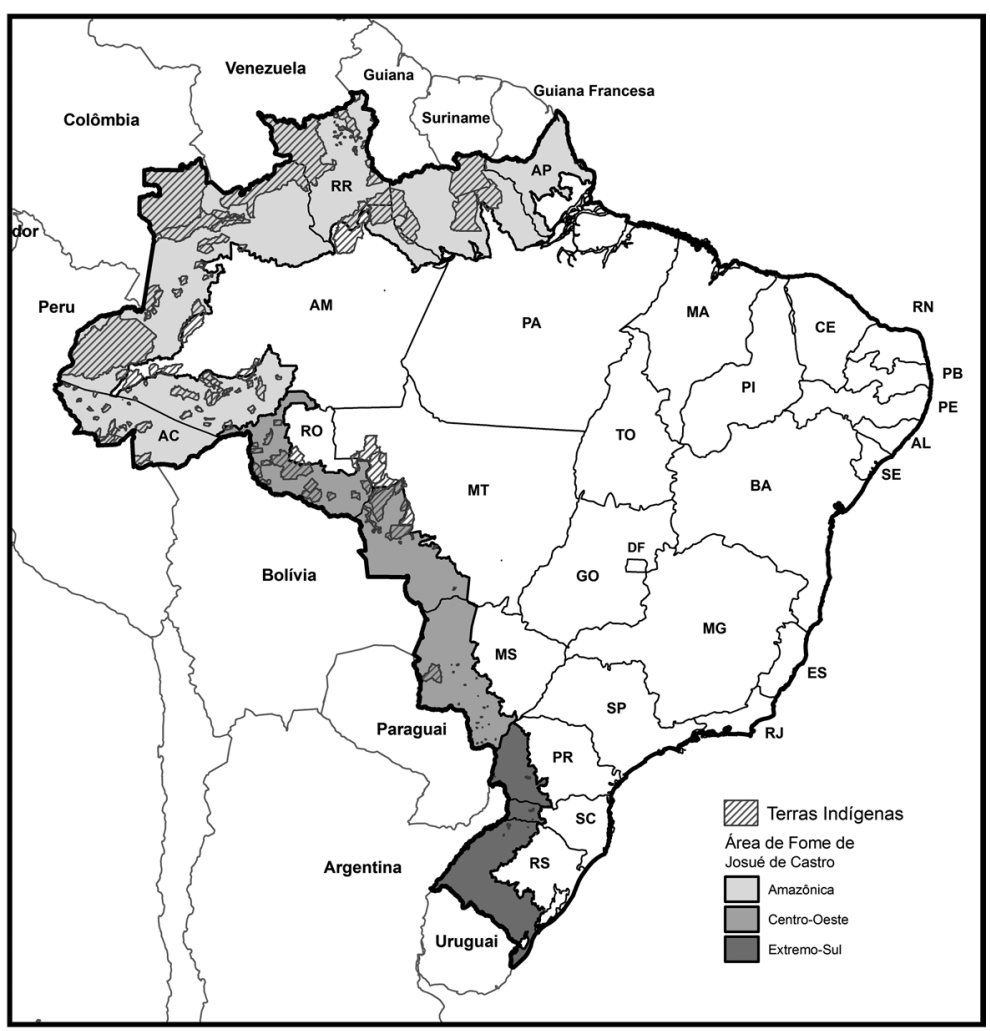

FIGURA 1 - Faixa de fronteira brasileira na América do Sul, dividida em três regiões culturais (Áreas de fome Amazônica, centro-oeste e extremo-sul). heterossexual, 15,8\% na categoria de transmissão de usuários de drogas injetáveis, $7,4 \%$ na categoria de transmissão ignorada e $5,4 \%$ nas outras categorias de transmissão (hemofilia, transfusão de sangue e vertical). A razão de sexo diminuiu de 3,7 em 1990, para 2,0 em 1995, para 1,5 em 2000 e para 1,4 em 2003. No que tange à escolaridade, 4,5\% nunca foram à escola, $25 \%$ declararam ter entre $1-3$ anos de escolaridade, $30,2 \%$ entre $4-7$ anos, $16,1 \%$ entre $8-11$ anos, 4,6\% acima de 12 anos e 15,2\% das notificações não apresentaram a informação sobre escolaridade.

A distribuição por faixa etária revelou que 5,4\% (428) dos casos notificados tinham até 13 anos, $13,9 \%$ (1.108) tinham entre $14-23$ anos, $65,7 \%$ (5.241) tinham entre $24-43$ anos, $14 \%$ (1.112) tinham entre $44-63$ anos e 1,1\% (85) tinham mais de 64 anos de idade. A distribuição de frequências na faixa etária de 14-43 anos por região cultural revelou 71,6\% de casos na faixa de fronteira extremo-sul, $19,7 \%$ na centrooeste e 8,7\% na Amazônica. Nesta faixa etária, 29,7\% dos casos apresentaram até três anos de escolaridade, na razão de sexo de 1,40;73,3\% dos casos foram notificados nas categorias de transmissão sexual, na razão de sexo de 1,1 (59,1\% na categoria heterossexual na razão de sexo de 0,7$)$, e 18,9\% dos casos foram notificados na categoria de usuários de drogas injetáveis na razão de sexo de 4,19; nesta faixa etária e na categoria de transmissão heterossexual, a razão de sexo foi 0,81 na faixa de fronteira Amazônica, 0,76 na centro-oeste e 


\begin{tabular}{|c|c|c|c|c|c|c|}
\hline \multirow[b]{3}{*}{ Doenças } & \multicolumn{6}{|c|}{ Regiões culturais } \\
\hline & \multicolumn{2}{|c|}{ Amazônica } & \multicolumn{2}{|c|}{ centro-oeste } & \multicolumn{2}{|c|}{ extremo-sul } \\
\hline & masc & fem & masc & fem & masc & fem \\
\hline Casos de aids notificados & 420 & 228 & 974 & 605 & 3.527 & 2.219 \\
\hline homens que fazem sexo com outros homens & 85 & --- & 108 & --- & 440 & --- \\
\hline bissexual & 63 & --- & 93 & --- & 298 & --- \\
\hline heterossexual & 181 & 207 & 442 & 517 & 1.392 & 1.867 \\
\hline usuários de drogas injetáveis/intravenosas & 27 & 7 & 143 & 41 & 850 & 191 \\
\hline hemofilia & 1 & --- & 4 & --- & 13 & --- \\
\hline transfusão & 3 & 4 & 6 & 15 & 11 & 7 \\
\hline vertical & 10 & 9 & 32 & 32 & 144 & 141 \\
\hline ignorado & 50 & 1 & 146 & --- & 379 & 13 \\
\hline Doenças associadas à inalação do agente & 126 & 51 & 324 & 139 & 1.025 & 372 \\
\hline tuberculose & 88 & 41 & 201 & 64 & 562 & 202 \\
\hline pneumonia Pneumocytis carinii & 46 & 14 & 130 & 81 & 466 & 179 \\
\hline micobacteriose & 9 & 3 & 11 & 3 & 76 & 28 \\
\hline histoplasmose & 8 & 2 & 8 & 3 & 26 & 10 \\
\hline Doenças associadas à ingestão contaminada & 82 & 32 & 106 & 57 & 357 & 168 \\
\hline criptococose & 11 & 2 & 25 & 6 & 110 & 30 \\
\hline criptosporidiose & 22 & 4 & 11 & 1 & 28 & 4 \\
\hline isosporidiose & 12 & 5 & 12 & 3 & 26 & 7 \\
\hline salmonelose & 2 & 1 & 1 & 3 & 30 & 17 \\
\hline toxoplasmose & 55 & 26 & 84 & 51 & 296 & 147 \\
\hline Doenças associadas ao contato interpessoal & 207 & 96 & 551 & 300 & 1.612 & 828 \\
\hline citomegalovirose & 11 & 2 & 21 & 12 & 67 & 33 \\
\hline herpes & 44 & 14 & 99 & 62 & 598 & 284 \\
\hline monilíase & 246 & 112 & 675 & 374 & 1.889 & 963 \\
\hline
\end{tabular}

0,67 na extremo-sul; nesta faixa etária e com a categoria de transmissão ignorada, houve 45 notificações na faixa de fronteira Amazônica (44 homens e 1 mulher), 109 na centro-oeste (todos homens) e 290 na extremo-sul ( 279 homens e 11 mulheres).

As doenças transmitidas pelo contato interpessoal foram observadas em 45,1\% dos casos notificados de AIDS, seguidas das doenças transmitidas pela inalação do agente etiológico $(25,6 \%)$ e das doenças transmitidas pela ingestão de água e/ou alimentos contaminados (10,1\%). Dentre os casos notificados pelos municípios da faixa de fronteira Amazônica, 46,8\% apresentaram doenças transmitidas pelo relacionamento humano, $27,3 \%$ de doenças transmitidas pela inalação do agente etiológico e 17,6\% de doenças transmitidas pela ingestão de água/alimentos contaminados; da faixa de fronteira centro-oeste, foram observados $53,9 \%, 29,3 \%$ e $10,3 \%$, respectivamente; na faixa de fronteira extremo-sul, foram $42,5 \%$, $24,3 \%$ e $9,1 \%$, respectivamente.

Dentre os casos de AIDS que apresentaram doenças oportunistas transmitidas pela inalação do agente etiológico, com idade entre 14-43 anos, residentes na faixa de fronteira Amazônica, 51,3\% foram notificados na categoria de transmissão heterossexual e 8,9\% na categoria de usuários de drogas injetáveis; dos residentes na faixa de fronteira centro-oeste houve $56,4 \%$ e $20,5 \%$, respectivamente, e na faixa de fronteira extremo-sul houve $46,9 \%$ e $29,7 \%$, respectivamente; a razão de sexo na faixa de fronteira Amazônica foi 0,49 na categoria de transmissão heterossexual e 3,67 na categoria de transmissão de usuários de drogas injetáveis; na faixa de fronteira centro-oeste, a razão foi 1,42 e 3,44, respectivamente, e na faixa de fronteira extremosul, 1,06 e 6,15, respectivamente.

Dos casos de AIDS com doenças oportunistas transmitidas pela ingestão de água e/ou alimentos contaminados, com idade entre 14-43 anos, residentes na faixa de fronteira Amazônica, 58,3\% notificações na categoria de transmissão heterossexual e 5,2\% na categoria de usuários de drogas injetáveis; na faixa de fronteira centrooeste houve $66,7 \%$ e $14,8 \%$, respectivamente, e na faixa de fronteira extremo-sul houve $53 \%$ e $21,5 \%$, respectivamente; a razão de sexo na faixa de fronteira Amazônica foi 1,07 na categoria de transmissão heterossexual e 4,00 na categoria de transmissão de usuários de drogas injetáveis; na faixa de fronteira centro-oeste, a razão foi 0,96 e 4,00, respectivamente, e na faixa de fronteira extremo-sul, foi 0,91 e 3,23, respectivamente.

Dos casos de AIDS com doenças oportunistas transmitidas pelo contato interpessoal, com idade entre 14-43 anos, residentes na faixa de fronteira Amazônica, 57,1\% foram notificados na categoria de transmissão heterossexual e $6,8 \%$ na categoria de usuários de drogas injetáveis; na faixa de fronteira centro-oeste houve $59,7 \%$ e $16,5 \%$, respectivamente, e na faixa de fronteira extremo-sul houve $53,4 \%$ e $25,5 \%$, respectivamente; a razão de sexo na faixa de fronteira Amazônica foi 0,97 na categoria de transmissão heterossexual e 2,60 
na categoria de transmissão de usuários de drogas injetáveis; na faixa de fronteira centro-oeste, a razão foi 0,97 e 3,21 , respectivamente, e na faixa de fronteira extremo-sul, 0,83 e 4,29, respectivamente.

A evolução da epidemia de AIDS e dos grupos de doenças oportunistas está apresentada por diagramas de séries temporais, por região cultural. As Figuras 2, 3 e 4 destacam a magnitude da epidemia de AIDS na faixa de fronteira extremo-sul, apresentando a evolução da incidência de doenças oportunistas transmitidas pela inalação do agente etiológico, de doenças transmitidas pela ingestão de água e/ou alimentos contaminados e de doenças transmitidas pelo relacionamento humano, respectivamente.

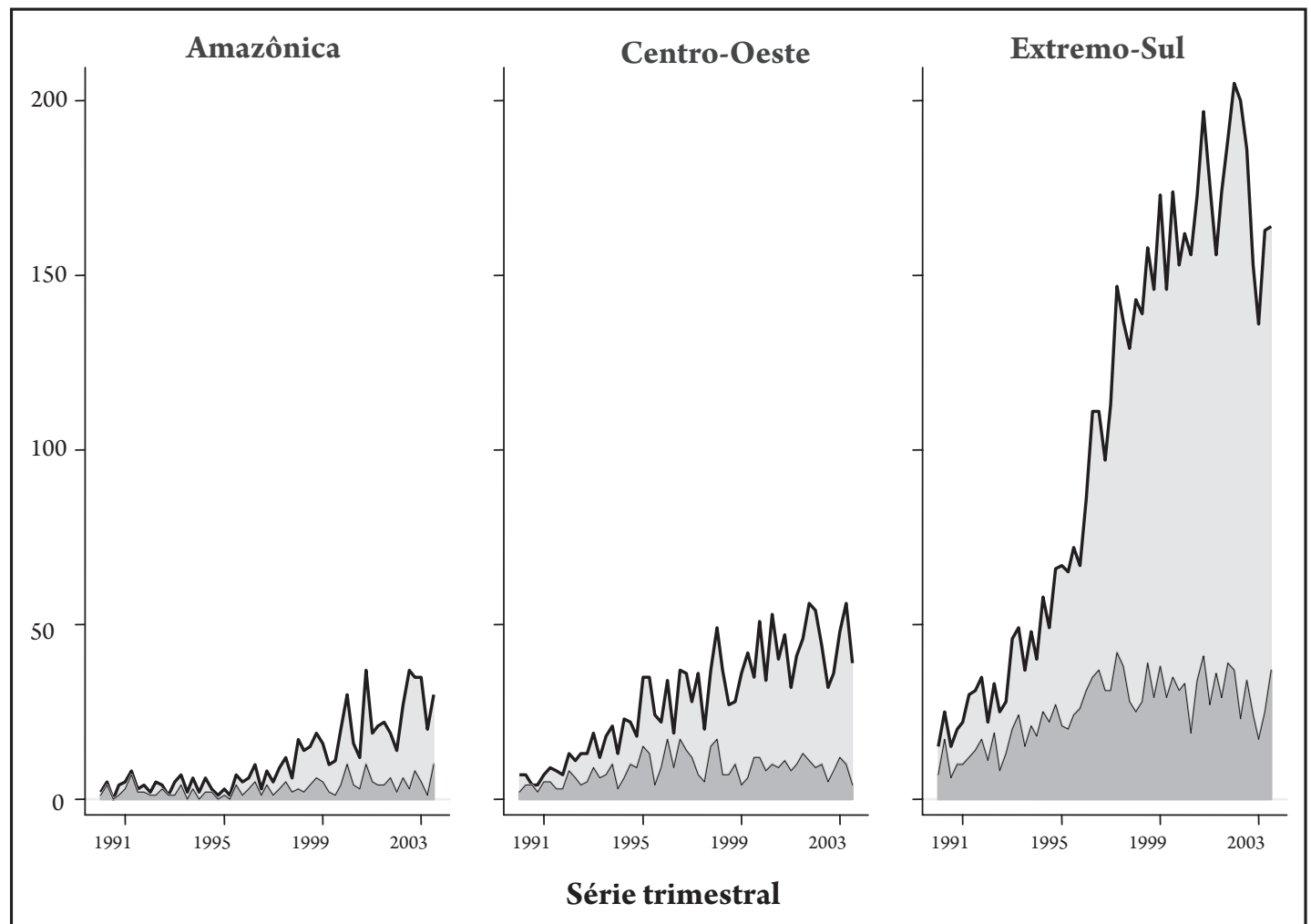

FIGURA 2 - Séries temporais da incidência de AIDS e das doenças transmitidas pela inalação do agente etiológico, nas três regiões culturais. PN-DST-AIDS. Brasil, 1990-2003.

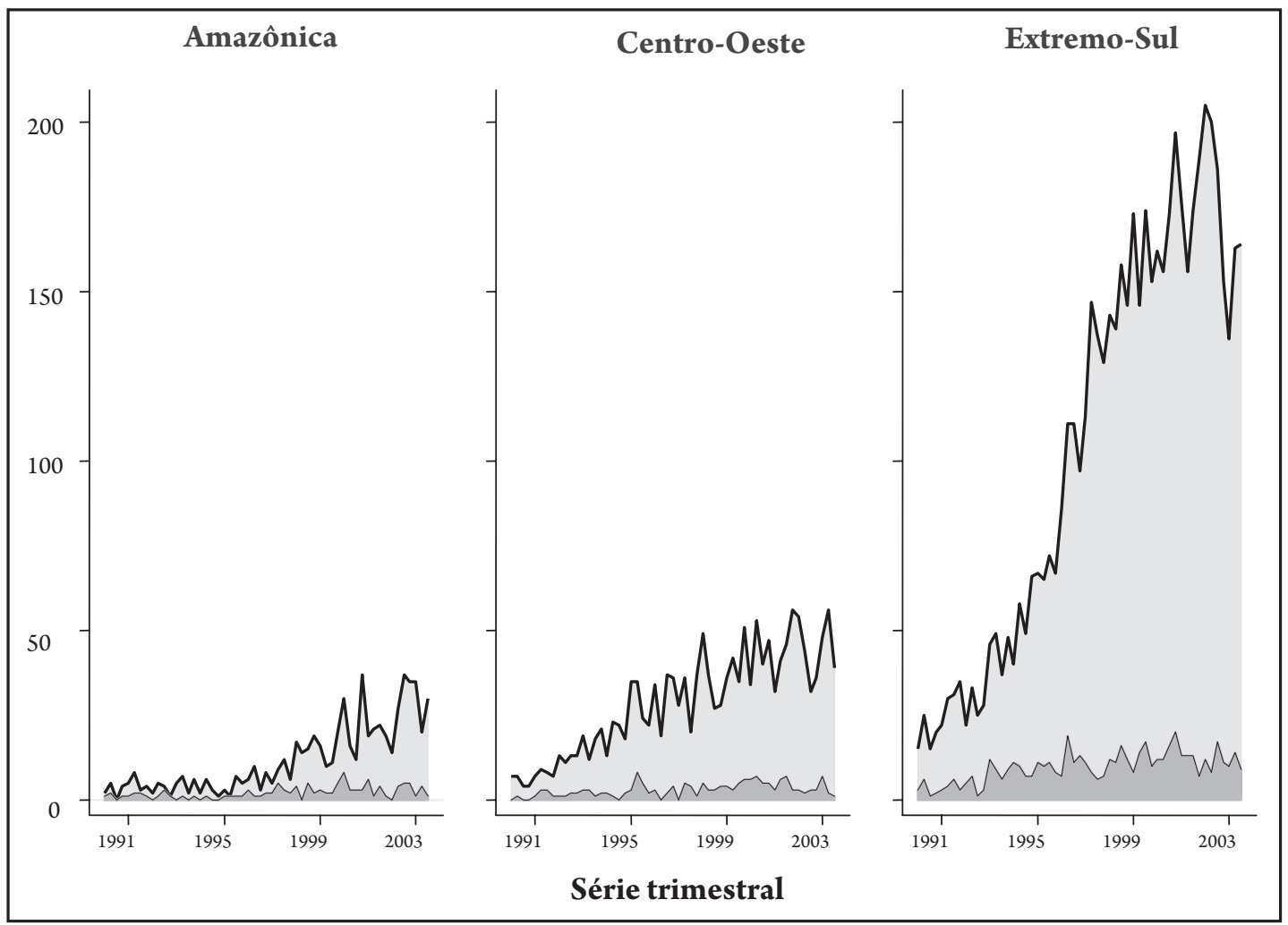

FIGURA 3 - Séries temporais da incidência de AIDS e das doenças transmitidas pela ingestão de água e/ou alimentos contaminados, nas três regiões culturais. PN-DST-AIDS. Brasil, 1990-2003. 


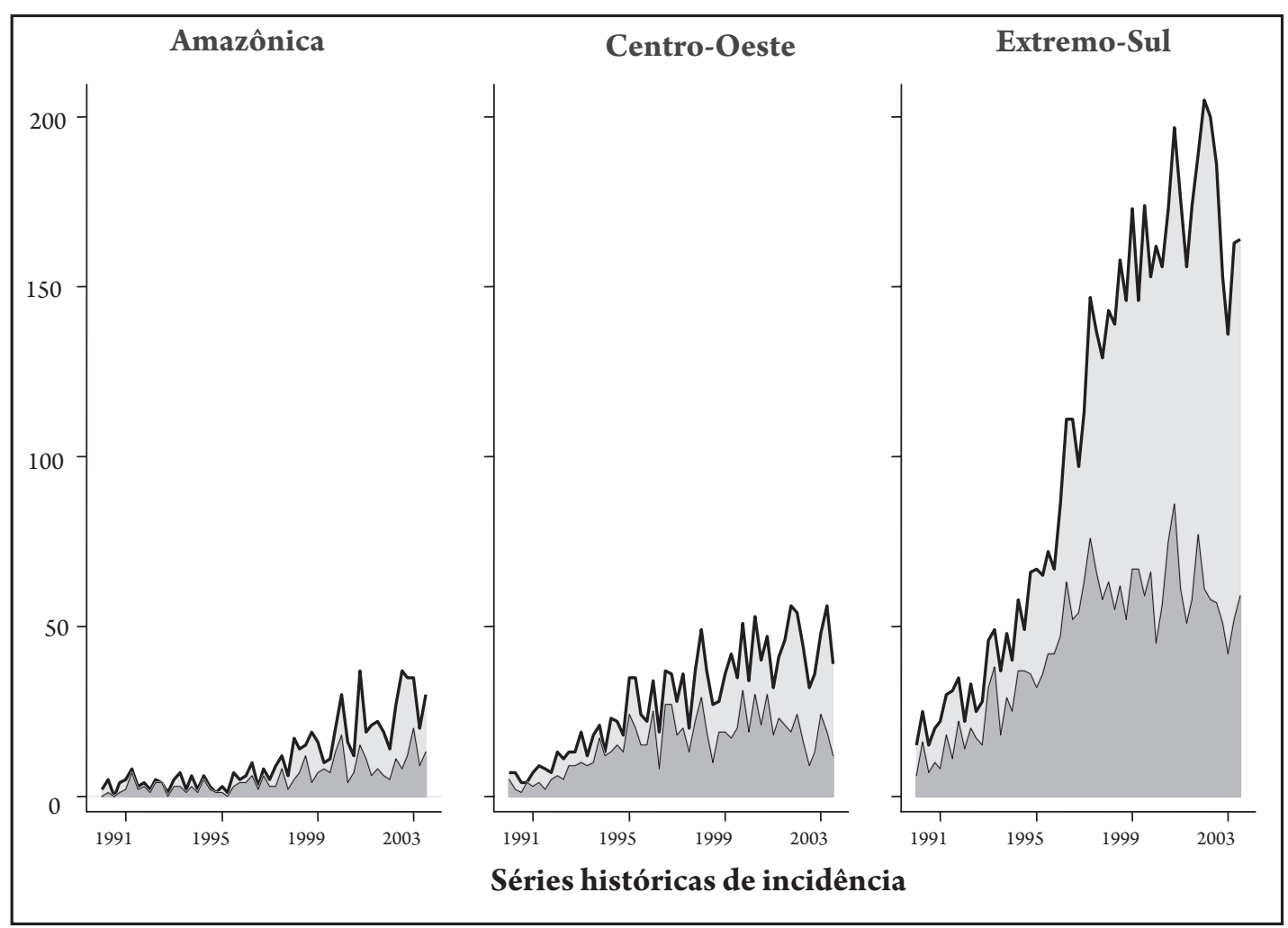

FIGURA 4 - Séries temporais da incidência de AIDS e das doenças transmitidas pelo contato interpessoal, nas três regiões culturais. PN-DST-AIDS. Brasil, 1990-2003.

\section{DISCUSSÃO}

A faixa de fronteira brasileira, tem sido associada à degradação do espaço geográfico ao longo de toda sua extensão territorial. Destacam-se os conflitos relacionados à ocupação dos espaços vazios ou de reservas indígenas (Figura 1), à exploração ilegal de madeira, à atividade de garimpo, à grilagem da terra e à posse da terra, na região fronteiriça da Guyana ${ }^{7}$; as atividades relacionadas às Forças Armadas Revolucionárias da Colômbia (FARC) e ao narcotráfico, que subjugam os moradores destas áreas e que provocam evasões em direção a Tabatinga, principalmente, ao Alto Solimões e a Manaus, na região fronteiriça da Venezuela e Colômbia ${ }^{5}$; as atividades do crime organizado, o tráfico humano, o contrabando, a exploração sexual infantil e o comércio ilegal de armas relacionadas à tríplice fronteira com a Argentina e o Paraguai ${ }^{3}$. A violência social dificulta o acesso à formação profissional, promove a desigualdade social, provoca o baixo desenvolvimento econômico, além de desestruturar os serviços de saúde, etc.

O estudo de incidência de AIDS na faixa de fronteira revelou a predominância da transmissão sexual, principalmente na categoria heterossexual, incluindo mulheres, jovens e pessoas com pouca, ou nenhuma, escolaridade, na rede de causalidade da doença. Os resultados deste estudo descreveram a incidência da AIDS em mulheres, na faixa etária de 14-43 anos e na categoria de transmissão heterossexual, e a incidência em homens, na mesma faixa etária e na categoria de transmissão de uso de drogas injetáveis. Sabe-se que a prática de sexo não-seguro e/ou do comércio sexual não-negociável, principalmente nas regiões caracterizadas pela iniquidade social e por violências, expõe mulheres jovens com pouca escolaridade, ou nenhuma, à epidemia de AIDS. Por outro lado, o padrão de exposição de homens adultos jovens na categoria de usuários de drogas injetáveis, principalmente nas faixas de fronteiras centro-oeste e extremo-sul, revela a participação nas atividades do narcotráfico, vendendo e/ou consumindo, contribuindo para o escoamento em direção aos pontos de exportação no litoral brasileiro.

O modelo de múltiplas causas e múltiplos efeitos fundamenta os resultados deste estudo, quando se considera a causalidade de doenças transmitidas pelo contato interpessoal e da AIDS. O estudo mostrou que as monilíases foram mais frequentes, na ocasião das notificações dos casos de AIDS, principalmente a candidíase oral e candidíase esofágica, provocada pela proliferação de Candida albicans e de Candida tropicalis causada pela deficiência imunológica; a herpes simplex e a herpes zoster foram também muito frequentes, pois o herpesvírus tem distribuição global. Dentre as doenças transmitidas pela inalação do agente etiológico, a tuberculose foi encontrada com maior frequência, considerando que a falta de equidade é determinante comum a ambas (AIDS e tuberculose); a tuberculose, por não ter sido efetivamente controlada pelas políticas públicas, é, hoje, reconhecida como doença negligenciada, cuja visibilidade reemergiu com a epidemia de AIDS, que, pelo abandono do tratamento sem alcançar a cura efetiva, tem gerado formas, potencialmente letais, pela resistência à quimioterapia; a pneumonia também foi bastante frequente. Das doenças oportunistas transmitidas pela ingestão de água e/ou alimentos contaminados, a toxoplasmose prevaleceu, sendo conhecida como a doença do gato, cuja transmissão ocorre pela ingestão de oocistos presentes no meio ambiente cosmopolita (solo, areia e latas de lixo contaminadas pelas fezes de gatos), pela ingestão de carne de porco e de carneiro cruas e pela transmissão transplacentária; a criptococose também foi bastante frequente nas faixas de fronteira centro-oeste e extremo-sul, cuja manifestação decorre do uso prolongado de corticosteróides, pois há resistência natural da raça humana ao Criptococcus, que é um fungo saprófito encontrado no solo, em frutas secas, cereais, nas árvores e em excrementos de aves, especialmente de pombos. Entretanto, na faixa 
de fronteira Amazônica, a criptosporidiose, que é transmitida por gatos e animais domésticos, e a isosporidiose foram mais frequentes que a criptococose.

A evolução temporal da AIDS revelou a magnitude da epidemia na faixa de fronteira extremo-sul, com aumento da incidência após 1995. O padrão temporal de incidência de casos com doenças oportunistas, transmitidas pela ingestão de água/alimentos contaminados, permaneceu baixo, quase inalterado, quando comparado à incidência de AIDS, nas três faixas de fronteira. $\mathrm{O}$ padrão longitudinal de incidência de casos com doenças transmitidas pela inalação do agente etiológico parece não ter alterado nas faixas de fronteira Amazônica e centro-oeste, mas, na faixa de fronteira extremo-sul houve um aumento discreto. $O$ padrão temporal de incidência das doenças transmitidas pelo relacionamento humano foi semelhante ao padrão evolutivo da epidemia de AIDS, apresentando um crescimento da incidência ao longo do tempo, nas faixas de fronteira Amazônica e centro-oeste; entretanto, na faixa de fronteira extremo-sul a incidência deste grupo de doenças oportunistas acompanhou a evolução de notificação de AIDS até meados de 1997, quando parece ter estabilizado. Em 2004, foram revisados os critérios Rio de Janeiro/Caracas e CDC modificado, que passaram a considerar a evidência laboratorial de contagem de linfócitos $\mathrm{T} \mathrm{CD}^{+}$(menor do que 350 células $/ \mathrm{mm}^{3}$ ).

O PN-DST/AIDS tem registrado algumas doenças transmitidas pelo contato com animais ou com reservatórios naturais, sustentando a teoria de Evgene Pavlovski. Várias das doenças indicativas de caso de AIDS ocorrem devido ao convívio do indivíduo com condições sanitárias insalubres, que podem expor os indivíduos imunodeprimidos às doenças infecciosas, quando convivendo próximos aos reservatórios naturais, como dengue, leishmaniose, brucelose, doença de Chagas, doença de Lyme, febre amarela, febre maculosa brasileira, filaríase, hantavirose, gripe, leptospirose, oncocercose, paracoccidioidomicose, peste, psitacose, raiva e tétano, apenas citando as doenças monitoradas pela Vigilância Epidemiológica. As doenças emergentes, principalmente aquelas vetorizadas pelas aves migratórias, como a febre do Nilo, a síndrome respiratória severa aguda e a gripe aviária, representam perigos potenciais, tanto para os pacientes com AIDS quanto para a difusão epidêmica entre a população em geral, considerando que as barreiras entre espécies podem ser rompidas, por mutantes ou por deficiência imunológica, desde que haja, necessariamente, o contato humano com os animas silvestres ou o contato de animais domésticos com animais silvestres. Há outras doenças oportunistas associadas à AIDS, cujas causas estão relacionadas às vulnerabilidades sociais, conhecidas como doenças negligenciadas, como a tuberculose e a hanseníase. Nestas, o contato ou a comunicação com indivíduo bacilífero é um determinante necessário, mas não suficiente, pois, em muitos casos, deve ocorrer a interação com fatores sociais para desencadear o processo patogênico, geralmente ligados às baixas condições de vida ou de inequidade/iniquidade. Tal raciocínio é compatível com a teoria de complexos patogênicos de Maximillian Sorré. O indivíduo com AIDS é mais susceptível às doenças emergentes e às doenças negligenciadas e poderiam ser acompanhados pelos serviços de saúde ou de vigilância epidemiológica, a partir do diagnóstico, como uma população vulnerável e sentinela, tanto na faixa de fronteira quanto em todo o território brasileiro.

Na região de fronteira, onde as dificuldades encontradas pelos serviços de saúde são enormes e prementes, o Sistema Integrado de Saúde na faixa de fronteira brasileira (SIS-fronteira) pretende contribuir com a melhoria e a organização dos serviços de saúde, principalmente a Vigilância em Saúde, e para a atuação do estado. A Secretaria de Vigilância em Saúde, do Ministério da Saúde, implantou a Rede de Laboratórios da Fronteira, buscando a maior rapidez do diagnóstico de doenças, incluindo o AIDS, presentes nos Municípios Oiapoque-AP, PacaraimaRR, Tabatinga-AM, Brasiléia-AC, Guarajá-Mirim-RO, Cáceres-MT, Ponta-Porã-MS, Foz do Iguaçu-PR, Dionísio Cerqueira-SC, São Borja-RS, Uruguaiana-RS e Santana do Livramento-RS. Entretanto, a distribuição geográfica dos Laboratórios Nível de Biossegurança 3 (NB-3), que são preparados para manipular agentes etiológicos de doenças emergentes, não contribui para tornar o serviço acessível pelo municípios da faixa de fronteira brasileira, principalmente nas faixas de fronteira Amazônica e centro-oeste. Constitui uma limitação deste estudo, além do questionamento sobre a exatidão dos diagnósticos das doenças oportunistas, lidar somente com as infecções oportunistas observadas no momento da notificação.

\section{AGRADECIMENTOS}

Ao Programa Nacional de Doenças Sexualmente Transmitidas e AIDS do Ministério da Saúde, pelo acesso ao banco de dados de notificação.

\section{CONFLITO DE INTERESSE}

Os autores declaram não haver nenhum tipo de conflito de interesse no desenvolvimento do estudo.

\section{REFERÊNCIAS}

1. Barcellos C, Peiter P, Rojas LI, Matida A. A geografia da AIDS nas fronteiras do Brasil. Campinas: Ministério da Saúde/CN DST/AIDS; Population Council e USAID; 2001.

2. Becker BK. Amazônia. São Paulo: Editora Ática; 1990.

3. Leonardi VPB. Violência e direitos humanos nas fronteiras do Brasil. Brasília: Paralelo 15; 2007.

4. Martin AR. Fronteiras e nações. São Paulo: Editora Contexto; 1989.

5. Oliveira MM. A mobilidade humana na triplica fronteira: Peru, Brasil e Colômbia Estud Avançados 2006; 20:183-196.

6. Peiter PC. A geografia da saúde na faixa de fronteira continental do Brasil na passagem do milênio. [doutorado]. [Rio de Janeiro]: Instituto de Geociências da Universidade Federal do Rio de Janeiro; 2005. 154p.

7. Rodrigues F. Migração transfronteiriça na Venezuela. Estud Avançados 2006; 20:197-207.

8. Sprandel MA. Brasileiros na fronteira com o Paraguai. Estud Avançados 2006; 20:137-156

9. Friedman SR, Kippax SC, Phaswana-Mafuya N, Rossi D, Newman CE. Emerging future issues in HIV/AIDS social research. AIDS 2006; 20:959-965.

10. Rodrigues-Jr AL, Castilho EA. A epidemia de AIDS no Brasil, 1991-2000: descrição espaço-temporal. Rev Soc Bras Med Trop 2004; 37:312-327.

11. Guimarães MDC. Estudo temporal das doenças associadas à AIDS no Brasil 1980-1999. Cad Saude Publica 2000; 16(supl 1):21-36.

12. Lima EJA, Pereira LE, Souza RP. Encefalite do Nilo Ocidental, nossa próxima epidemia? Epidemiol Serv Saude 2003; 12:7-19.

13. Ostroff SM. Emerging infectious diseases 1997-1998: the role of molecular epidemiology. Mem Inst Oswaldo Cruz 1999; 94:1-3.

14. Castro J. Geografia da fome. Rio de Janeiro: Antares: Achiamé; 1980.

15. Chin J. Manual de controle das doenças transmissíveis. Porto Alegre: Editora Artmed; 2002

16. Medronho RA, Carvalho DM, Bloch KV, Luiz RR, Werneck GL. Epidemiologia. São Paulo: Editora Atheneu; 2002.

17. Ministério da Saúde. Secretaria de Vigilância em Saúde. Programa Nacional de DST e AIDS. Critérios de definição de casos de AIDS em adultos e crianças. Ministério da Saúde, Secretaria de Vigilância em Saúde, Programa Nacional de DST e AIDS: Ministério da Saúde; 2003. 